\title{
Universal Diagrams for TE Waves Guided by Thin Films Bounded by Saturable Nonlinear Media
}

\author{
Juan P. Torres and Lluís Torner, Member, IEEE
}

\begin{abstract}
We show that universal $V-b$ diagrams provide a powerful tool when analyzing the stationary waveguiding properties of the TE waves guided by a thin film bounded by a saturable nonlinear substrate or cladding. For a wide class of nonlinearities, the allowed and forbidden regions of these diagrams, for stationary guided propagation to occur, display a universal pattern, the marginal loci separating different allowed regions from the forbidden ones being simple functions of only the asymmetry measure of the waveguide and the saturation value of the nonlinear permitivity. Relevant information for device design purposes is summarized on a few diagrams, so general waveguiding properties can be immediately read-off from them, and threshold power-independent values of the normalized thickness of the waveguide for a particular kind of guided waves to be allowed are obtained. Qualitative information concerning both the guided power and the stability of guided waves is also included in the diagrams.
\end{abstract}

\section{INTRODUCTION}

$\mathrm{D}$ SPERSION properties of nonlinear TE-polarized waves guided by planar optical waveguides with a nonlinear substrate or cladding have been extensively studied during the last few years [1]-[7], so now main features concerning stationary guided phenomena are well established. This waveguiding structure plays a crucial role in various all-optical switching devices [8], [9], such as the nonlinear directional coupler [10], [11] or the soliton coupler [12], and in upper and lower threshold power devices [13]. In addition to the Kerr-like behavior, more general cases, such as high-order nonlinearities [14]-[16] and saturable media [17]-[18], have been investigated in order to take into account some departures from ideality which arise in real materials. However, due to the powerdependent nature of the above mentioned dispersion relations and, also, to the great number of waveguide parameters involved, it is difficult to identify general rules concerning global waveguiding characteristics. For instance, we refer to figures of merit of a particular guiding structure, to cutoff conditions for the different type of possible stationary nonlinear guided waves or to critical values of the various waveguide parameters which partition parameter space into regions of similar behavior. Our aim here addresses this point. Knowledge of this information

\footnotetext{
Manuscript received February 24, 1992; revised July 10, 1992. This work was supported by the Comisión Interministerial de Ciencia y Tecnología of the Spanish Government under Grant TIC 92-0094 C02-02.

The authors are with the Department of Signal Theory and Communications, Polytechnic University of Catalonia, P.O. Box 30002, 08080 Barcelona, Spain.

IEEE Log Number 9206529
}

is crucial for the device design, since it establishes the conditions in parameter space for the waveguide being able to perform the envisaged function (e.g., power limiting, thresholding or bistability). Ultimately, it allows identification of regions in parameter space yielding waveguides whose properties are less sensitive to fabrication tolerances.

In the case of an ideal Kerr-like medium, in a previous paper [19] we have shown that a great deal of information can be read-off from the well-known $V-b$ diagrams. Here $b$ stands for the normalized effective index of guided waves and $V$ is the normalized thickness of the waveguide [20]. Our goal in this paper is to show that these diagrams provide also a very powerful tool when considering other than Kerr-like media. We will point out that, for a wide class of nonlinearities, the allowed and forbidden regions of the $V-b$ plane for stationary guided propagation to occur display a universal pattern. From this plot, general results can be immediately obtained without solving the nonlinear differential problem. For instance, we will be able to identify critical threshold, power-independent values of the normalized thickness for a particular kind of guided waves to be allowed and to obtain the expressions of the marginal loci in the parameter space separating the allowed from the forbidden bands.

All optically nonlinear materials exhibit saturation, in addition to absorption. For all-optical devices, large saturation values of the nonlinear refractive index, while low absorption, are desirable. First goal has been achieved, e.g., in liquid crystals, thus existence of nonlinear guided waves has been demonstrated in a MBBA-cladded planar waveguide [21]. Smaller intensity-dependent optically induced refractive index changes, but large enough for switching applications, have also been demonstrated in GaAs-AlGaAs multiple-quantum-well (MQW) structures operating near a resonance. Low absorption has been demonstrated using nonresonant nonlinearities in semiconductor doped glasses [22]. Likewise, recent measurements of two-photon absorption coefficient have shown that unwanted effects of absorption can be also avoided in GaAs-AlGaAs MQW's operating below one-half the bandgap [23]. Here we will assume low absorption operation regime and, in order to obtain universal results, we will deal with both non-specified self-focusing and self-defocusing media, having a typical two-level saturable behavior. The starting point of our analysis is the generalized dispersion relation for nonlinear guided waves 
derived by Langbein, Lederer, and Ponath [8]. The same authors [17] have investigated the behavior of nonlinear guided waves in saturable media from a point of view similar to the general approach discussed here, so we will recover some of their results.

This paper is organized as follows. Section II contains the background of our approach. It is devoted to the introduction of the normalized parameters and to a brief revision of the different type of possible field solutions corresponding to the roots of the normalized nonlinear dispersion equation. In Section III the allowed and forbidden bands in the universal $V-b$ plane for stationary guided propagation to occur are identified and classified. The expressions of the marginal loci separating different regions are given for both self-focusing and self-defocusing cases. In Section IV the critical values of the normalized thickness emerging from the pattern of the allowed regions are calculated. The limiting value of the guided wave power at the marginal loci separating the allowed from the forbidden bands is discussed in Section V. In Section VI we focus on the normalized dispersion relation for the guided wave power. The marginal locus in the $V-b$ plane indicating the region in parameter space where the guided power increases monotonically with the effective index of the allowed stationary guided solutions is calculated and analyzed, and its relevance concerning the stability of the nonlinear guided waves is discussed. Finally, in Section VII we shall emphasize our main results.

\section{Normalized Dispersion Equation}

We will consider a three layer dielectric structure (Fig. 1) which consists of a thin optically linear film of thickness $D$ and refractive index $n_{f}$, a linear cover with refractive index $n_{c}$ and a nonlinear media forming the substrate whose intensity-dependent dielectric function is

$$
\epsilon_{s}=\epsilon_{0}\left[n_{s}^{2}+\epsilon^{\mathrm{NL}}\left(E^{2}, \mu_{s}\right)\right]
$$

with $\epsilon_{0}$ being the free-space permitivity. When considering saturable permitivities, for low-powers $\epsilon^{\mathrm{NL}}$ behave as it does for Kerr-like media, but saturates for high powers. Since we are looking for universal results, instead of taking a particular expression for $\epsilon^{\mathrm{NL}}$, we will consider an unspecified function, positive defined in the self-focusing case and negative defined in the self-focusing one, with a typical saturable behavior. For the seek of clarity, what must be exactly understood for this last restriction will be clear a bit later $[c f$. paragraph after (7)]. Generally speaking, $\epsilon^{\mathrm{NL}}$ will depend on a set of parameters that we have represented by $\mu_{s}$. Here we will restrict to the case $n_{f}>$ $n_{s} \geq n_{c}$, although the analysis holds for a general case. However, we note that due to the nonlinearity, both cases $n_{s}>n_{c}$ and $n_{s}<n_{c}$ must be considered separately. We look for TE-polarized stationary guided waves so that the electric field $\mathcal{E}(\vec{r}, t)$ would write

$$
\varepsilon_{y}(z, t)=\frac{1}{2} E(z) \exp \left[j\left(w t-k_{0} N x\right)\right]+\text { c.c. }
$$

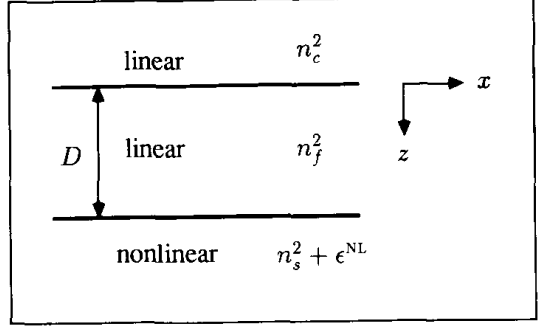

Fig. 1. Waveguide structure.

where $k_{0}$ is the free space wavenumber and $N=\beta / k_{0}$ the effective modal index. Finally, from now on we will use the dimensionless coordinate $\xi=z / D$ and the dimensionless field function $\Psi(\xi)=E(\xi) / E_{0}$, with $E_{0}$ being a constant to be specified.

The dispersion equation for the TE waves guided by a nonlinear waveguide such as the one described above has been obtained by Langbein, Lederer, and Ponath [8]. In terms of the well-known normalized parameters: the normalized thickness $(V)$ and asymmetry measure $(a)$ of the waveguide

$$
V=k_{0} D \sqrt{n_{f}^{2}-n_{s}^{2}}, \quad a=\frac{n_{s}^{2}-n_{c}^{2}}{n_{f}^{2}-n_{s}^{2}}
$$

and the normalized effective index

$$
b=\frac{N^{2}-n_{s}^{2}}{n_{f}^{2}-n_{s}^{2}}
$$

the dispersion relation writes

$$
\begin{aligned}
V \sqrt{1-b}= & m \pi+\tan ^{-1} \sqrt{\frac{a+b}{1-b}} \\
& +(-1)^{M} \tan ^{-1} \sqrt{\frac{b-d}{1-b}}
\end{aligned}
$$

for $b<1$, and

$$
V \sqrt{b-1}=\tanh ^{-1} \sqrt{\frac{b-1}{b-d}}-\tanh ^{-1} \sqrt{\frac{b-1}{a+b}}
$$

for $b \geq 1$. In (5), $M=0$ for field solutions having no maximum in the substrate region and $M=1$ otherwise, and $m$ stands for the number of nodes of the field in the film region. Finally $d$, which can be related to the value of the field at the film-substrate interface, is given by $d=$ $\left.f\left(\Psi_{s}\right)\right|_{\xi=1}$, where $f\left(\Psi_{s}\right)$ is an adimensional function defined as

$$
f\left(\Psi_{s}\right)=\frac{1}{n_{f}^{2}-n_{s}^{2}} \frac{1}{\Psi_{s}^{2}} \int_{0}^{\Psi_{s}^{2}} \epsilon^{\mathrm{NL}}\left(\Psi_{s}^{2}, \mu_{s}, E_{0}\right) d \Psi_{s}^{2} .
$$

We will restrict to nonlinear permitivities $\epsilon^{\mathrm{NL}}$ for which the function $f\left(\Psi_{s}\right)$ increases (self-focusing case) or decreases (self-defocusing media), monotonically. This condition is not essential but avoids the existence of dou- 
ble solutions of (5) and (6), with equal $b$ but corresponding to different values of the guided wave power, which should modify some conclusions from the paper. It is worth to notice that most of the saturable, as well as nonsaturable permitivities suggested in the literature satisfy the above requirement.

For given values of $V$ and $a,(5)$ and (6) give us the normalized effective index of a $\mathrm{TE}_{m}$ guided wave as a function of $M$ and $d$. However, for a fixed value of $a$, there are some regions in the $V-b$ plane which cannot be reached with the physically possible values of $d$. Also, when solving (5) and (6) for $b$, it should be verified that the obtained values of the normalized effective index render to field solutions which satisfy the nonlinear wave equation, so the shape of the corresponding field solution satisfy the implicit condition included into the taken value of $M$. Thus, allowed and forbidden regions in the $V-b$ plane will exist. The point is that these regions can be identified and classified without solving the dispersion equation.

III. Allowed and Forbidden Regions in the $V-b$ Diagrams

Let us start with the nonlinear wave equation. In the substrate, it states

$$
\frac{d^{2} \Psi_{s}(\xi)}{d \xi^{2}}-V^{2}\left[b-\frac{\epsilon^{\mathrm{NL}}\left(\Psi_{s}^{2}(\xi), \mu_{s}, E_{0}\right)}{n_{f}^{2}-n_{s}^{2}}\right] \Psi_{s}(\xi)=0 .
$$

Since the substrate is assumed to be a lossless medium, $\Psi_{s}(\xi)$ is real, thus the first integral of this equation is found to be

$$
\Phi_{s}=\left(\frac{d \Psi_{s}(\xi)}{d \xi}\right)^{2}=V^{2}\left[b-f\left(\Psi_{s}\right)\right] \Psi_{s}^{2}(\xi) .
$$

In the case of a self-focusing medium $f\left(\Psi_{s}\right)$ is a positive defined function, bounded by a maximum value $f_{\max }$ if $\epsilon^{\mathrm{NL}}$ is bounded too. Since $f\left(\Psi_{s}\right)$ is assumed to increase monotonically, $f_{\max }$ will always be reached for $\Psi_{s} \rightarrow \infty$. For a Kerr-like nonlinear medium one has $f_{\max }=\infty$. When considering self-defocusing media, $f\left(\Psi_{s}\right)$ is a negative defined function.

Next we are going to analyze is the shape of the first integral $\Phi_{s}$ [8]. As a function of $\Psi_{s}, \Phi_{s}$ will show two different shapes depending on the value of $b$ and on the sign of $f\left(\Psi_{s}\right)$, as can be seen in Fig. 2. In the case of a self-focusing medium, when $b<f_{\max }$ there is a nonzero value of $\Psi_{s}$ which makes $\Phi_{s}=0$, so the electric field may show a local or global maximum at the substrate region $(\xi>1)$. This situation corresponds to the roots of (5) having $M=1$. On the contrary, when $b>f_{\max }$ no $\max$ imum of the electric field will occur in the substrate region, since $\Phi_{s}=0$ holds only when $\Psi_{s}=0$. This situation, which also occurs in the case of a self-defocusing medium for any value of $b$, corresponds to $M=0$. Electric field solutions having no maximum in the substrate

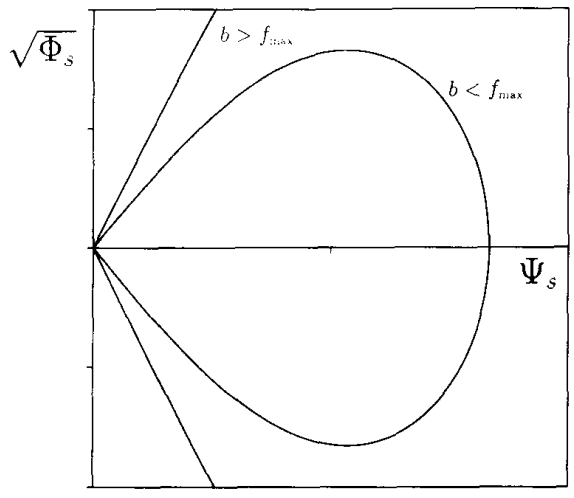

Fig. 2. Phase diagram pattern.

( $M=0$ ) correspond to the so-called pure guided waves (PGW). When considering a self-defocusing medium all guided waves correspond to this category, whereas for a self-focusing medium both situations $M=0,1$ are possible. For $M=1$ there are two different cases: when $b<$ 1 , the electric field in the film will be expressed in terms of trigonometric functions, whereas when $b>1$ the field in the film will be a combination of hyperbolic functions. In the first case, the electric field shape corresponds to a bulged guided wave (BGW) and in the second one to a surface wave (SW) [4], [6]. In view of the above considerations we are going to identify the allowed and forbidden regions in the $V-b$ plane in order to a stationary guided solution occur. Key points run as follows:

$A$. The value of $d$ is bounded. For a self-defocusing medium one has $f_{\min } \leq d \leq 0$, and in the self-focusing case $0 \geq d \geq d_{\max }$, with $d_{\max }=\min \left\{f_{\max }, 1\right\}$. This last condition follows from the boundary conditions at the film-substrate and film-cover interfaces.

$B$. Not all roots of (5) and (6) having $M=1$ are possible. This condition comes from the fact that for a selffocusing medium, when $b>f_{\max }$ no maximum of the electric field is allowed in the substrate region. Therefore, the corresponding value of $b$ is forbidden.

In Figs. 3-5 we have plotted the allowed and forbidden regions in the $V-b$ plane for guided propagation to occur, coming from items $A$ and $B$. Figs. 3 and 4 correspond to a self-focusing medium and Fig. 5 to a self-defocusing one. Let us focus now on Fig. 3. In this case, the marginal loci separating the allowed from the forbidden regions come from the conditions $\{d=0 ; M=0,1\}$, and $\{b=$ $\left.f_{\max } ; M=1\right\}$ in (5) and (6). In Fig. 4 one has $f_{\max }<1$, and the marginal loci come from the conditions $\{d=0$; $M=0,1\},\left\{d=f_{\max } ; M=0\right\}$ and $\left\{b=f_{\max } ; M=1\right\}$. In both cases, condition $d=0$ yields critical values of the normalized effective index which are referred to as $b_{c+}$ and $b_{c-}$, accordingly to the corresponding value of the $\operatorname{sign}(-1)^{M}$. A similar expression to the one yielding $b_{c+}$, holds for $d=f_{\max }$. In this case the associated values of $b$ are referred to as $b_{c}$. Finally, the condition $b=f_{\max }$ defines a straight horizontal line in the $V-b$ plane whose values of $b$ are referred to as $b_{c f}$. 


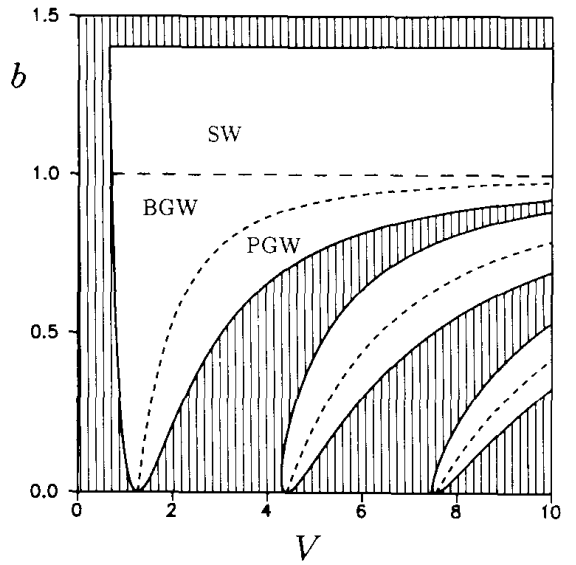

Fig. 3. Allowed and forbidden bands for stationary TE guided propagation in a waveguide with a self-focusing saturable bounding medium having $f_{\max }$ $>1$. PGW: pure guided waves; BGW: bulged guided waves; $S W$ : surface waves. Dashed area: forbidden region. Here $a=10$.

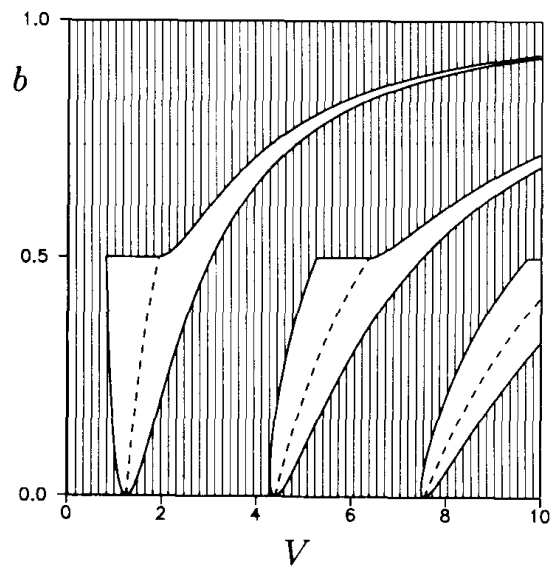

Fig. 4. Same as in Fig. 3 but for the strong saturation regime: $f_{\max }<1$.

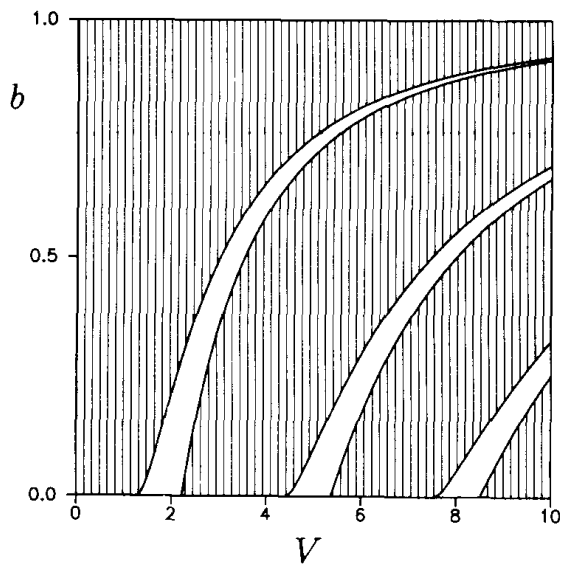

Fig. 5. Allowed and forbidden bands in the case of a self-defocusing medium.

Allowed bands subdivide into three regions, each one corresponding to the different type of possible guided wave solutions. When $f_{\max }>1$ all PGW, BGW and SW are allowed, the actual occurrence of one of them depending on the total amount of guided power and on the excitation conditions. In the opposite case, $f_{\max }<1$, only PGW and BGW remain possible [17]. Surface waves occur above the line $b=1$ and the loci in the $V-b$ plane separating pure from bulged guided wave solutions come from the condition $d=b$. This is the value of the normalized effective index (which will be denoted by $b_{c 0}$ ) at which a maximum of the electric field enters the substrate. According to (5), $b_{c 0}$ is given by

$$
V \sqrt{1-b_{c 0}}=m \pi+\tan ^{-1} \sqrt{\frac{a+b_{c 0}}{1-b_{c 0}}} .
$$

In the case of a self-defocusing medium only PGW can take place, and the marginal loci separating the allowed from the forbidden regions are given by $b_{c+}, b=0$ and $b_{c d}$. This former critical value corresponds to the limit $\{d$ $\left.=f_{\min } ; M=0\right\}$ in (5).

\section{Critical Normalized Thicknesses}

One of the main usefulness of recognizing allowed and forbidden bands in the $V-b$ plane, comes from the fact that it enables us to identify threshold, power-independent values of the normalized thickness $V$ for the different kind of guided solutions to occur. This fact can be clearly seen in Figs. 3-5. The important result is that these threshold $V$ values depend only on the asymmetry measure of the waveguide and on $f_{\max }$ (or $f_{\min }$ ).

First, we will pay attention to the $\mathrm{TE}_{0}$ wave on a selffocusing medium with $f_{\max }>1$. In this case, Fig. 3 shows that when the normalized thickness is greater than the lowpower cutoff thickness $\left(V_{L, \text { cutoff }}^{(m)}=m \pi+\tan ^{-1} \sqrt{a}\right)$ the three types of guided solutions are possible. However, from Fig. 3 we conclude that for $V<V_{L \text {,cutoff }}^{(0)}$ pure guided waves become no longer possible and two important characteristic normalized thicknesses arise: $V_{c s}$ and $V_{\text {cutoff }}^{(0)}$. The first one $V_{c s}$, is obtained by taking the limit $b_{c-}=1$ in (6). One gets

$$
V_{c s}=1-\frac{1}{\sqrt{1+a}} .
$$

When $V$ is greater than $V_{c s}$ but smaller than the low-power cutoff thickness, both BGW and SW are possible. Below $V_{c s}$, when $V$ lies in the range $V_{\text {curoff }}^{(0)}<V<V_{c s}$, only surface waves are possible. Finally, below $V_{\text {cutof }}^{(0)}$ stationary guided propagation cannot occur, for any value of the guided power and of the waveguide parameters. This threshold value of the normalized thickness for a guided solution to exist is obtained by taking the limit $b_{c-}=f_{\max }$ in (6). One arrives at

$$
\begin{aligned}
V_{\text {cutoff }}^{(0)}= & \frac{1}{\sqrt{f_{\max }-1}} \tanh ^{-1} \\
& \cdot \frac{\sqrt{f_{\max }-1}\left(\sqrt{a+f_{\max }}-\sqrt{f_{\max }}\right)}{1-f_{\max }+\sqrt{a+f_{\max }} \sqrt{f_{\max }}} .
\end{aligned}
$$


When $f_{\max } \gg 1$ (Kerr-like behavior), $V_{\text {cutoff }}^{(0)} \rightarrow 0$. Also, in the case of a symmetric waveguide $(a=0)$, both $V_{\text {cutoff }}^{(0)}$ and $V_{c s}$ vanishes, so there is no cutoff and all types of guided solutions are allowed in the waveguide for any value of $V$.

Let us turn now to a self-focusing medium with $f_{\max }<$ 1. In this case, only PGW and BGW are possible and there exist three critical values of the normalized thickness: $V_{\text {cutoff }}^{(0)}, V_{c p}^{(m)}$ and the linear cutoff thickness $V_{L \text {, cutoff. }}^{(m)} V_{\text {cutoff }}^{(0)}$ is obtained by taking $b_{c-}=f_{\max }$, and $V_{c p}^{(m)}$ comes from the substitution of $\left\{d=b=f_{\max } ; M=0\right\}$, both in (5). One gets

$$
\begin{aligned}
V_{\text {cutoff }}^{(0)}= & \frac{1}{\sqrt{1-f_{\max }}} \tan ^{-1} \\
& \cdot \frac{\sqrt{1-f_{\max }}\left(\sqrt{a+f_{\max }}-\sqrt{f_{\max }}\right)}{1-f_{\max }+\sqrt{a+f_{\max }} \sqrt{f_{\max }}}
\end{aligned}
$$

and

$$
V_{c p}^{(m)}=\frac{1}{\sqrt{1-f_{\max }}}\left[m \pi+\tan ^{-1} \sqrt{\frac{a+f_{\max }}{1-f_{\max }}}\right] .
$$

Concerning higher order TE waves, we realize that when $f_{\max }>1$ the corresponding allowed and forbidden regions are the same as for the ideal Kerr-like case. Thus, both PWG and BGW are possible for any value of $V$ greater than $V_{L \text {,cutoff }}^{(m)}$. However, when $f_{\max }<1$, above $V_{c p}^{(m)}$ only PGW remain possible again. In both cases, below the low-power cutoff thickness only BGW are allowed and there is a new threshold value for guided propagation to occur: $V_{\text {cutoff }}^{(m)}$. This critical value, which holds as well in the Kerr-like limit, is a function of $a$ defined by the expression

$$
\left.\frac{d b_{c-}}{d V}\right|_{V=V_{\text {cutoff }}^{(n) m \rightarrow \infty}}
$$

for $m \geq 1$. This is a transcendental equation, so it must be solved numerically. The obtained values for $m=1,2$ are shown in Fig. 6 as a function of $a$. We conclude from this plot that $V_{L \text {,cutoff }}^{(m)}=V_{\text {cutoff }}^{(m)}$ for symmetric waveguides, $V_{L, \text { cutoff }}^{(1)}-V_{\text {cutoff }}^{(1)} \simeq 0.11$ when $a>1$, and that smaller corrections occur for higher-order solutions.

Now, we will look at self-defocusing media. As shown in Fig. 5 , in this case below $V_{L \text {,cutoff }}^{(m)}$ stationary guided propagation cannot occur. Above $V_{L \text {,cutoff }}^{(m)}$ another critical value arises. It is found by making $b_{c d}=0$. One obtains

$$
V_{c d}^{(m)}=m \pi+\tan ^{-1} \sqrt{a}+\tan ^{-1} \sqrt{\left|f_{\min }\right|} .
$$

Above $V_{c d}^{(m)}$ the minimum allowed value of the normalized effective index is $b_{c d}$ whereas when $V_{L \text {,cutoff }}^{(m)}<V<V_{c d}^{(m)}$ one has $0<b<b_{c+}$. The meaning of $V_{c d}^{(m)}$ in terms of the guided power will render clear later on. Tables I-III summarize the main results obtained in this section.

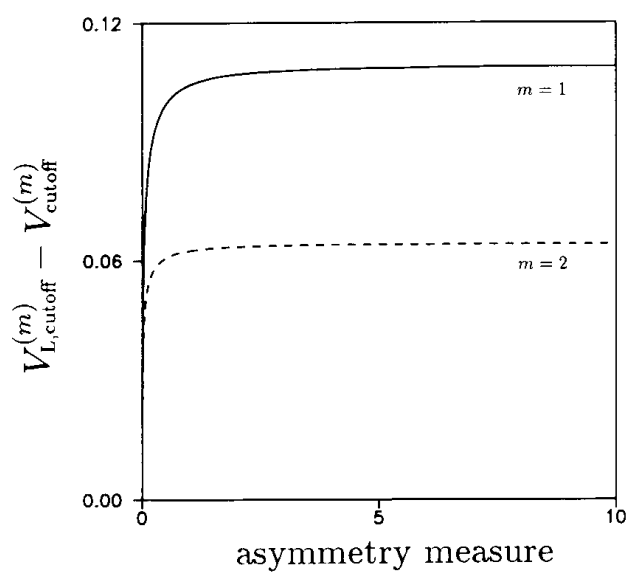

Fig. 6. Difference between the low-power normalized cutoff thickness and the threshold value of the normalized thickness for a stationary high-order TE guided wave to exist, $V_{\text {cutoff }}^{(m)}$.

TABLE I

Threshold Values of the Normalized Thickness for the Different Kind of Stationary TE 0 Guided Waves to be Allowed, in a Waveguide with a Self-Focusing Saturable Bounding Medium HAVING $f_{\max }>1$

\begin{tabular}{lccc}
\hline & PGW & BGW & SW \\
\hline$V<V_{\text {cutoff }}^{(0)}$ & NO & NO & NO \\
$V_{\text {cutoff }}^{(0)}<V<V_{c s}$ & NO & NO & YES \\
$V_{c s}<V<V_{L, \text { cutoff }}^{(0)}$ & NO & YES & YES \\
$V_{L, \text { cutoff }}^{\text {(0) }}<V$ & YES & YES & YES \\
\hline
\end{tabular}

TABLE II

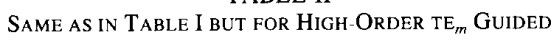
WAVES

\begin{tabular}{lcc}
\hline & PGW & BGW \\
\hline$V<V_{\text {cutoff }}^{(m)}$ & NO & NO \\
$V_{\text {cutoff }}^{(m)}<V<V_{L \text {.culoff }}^{(m)}$ & NO & YES \\
$V_{L, \text { cutoff }}^{(m)}<V$ & YES & YES \\
\hline
\end{tabular}

TABLE III

SAme AS IN TABLes I AND II BUt For the STRONG Saturation Regime: $f_{\max }<1$

\begin{tabular}{lcc}
\hline & PGW & BGW \\
\hline$V<V_{\text {culoff }}^{(m)}$ & NO & NO \\
$V_{\text {cuttoff }}^{(m)}<V<V_{L, \text { cutoff }}^{(m)}$ & NO & YES \\
$V_{L, \text { cutoff }}^{(m)}<V<V_{c p}^{(m)}$ & YES & YES \\
$V_{c p}^{(m)}<V$ & YES & NO \\
\hline
\end{tabular}

\section{On the Guided Power}

Our aim in this section is to obtain the limiting values of the guided power at the marginal loci in the $V-b$ plane separating the allowed from the forbidden regions. Table IV will collect the main conclusions. In terms of the adimensional field $\Psi(\xi)$, the power carried by a guided 
TABLE IV

Limiting Values of the Guided Power Flow at the Marginal Loci in the $V-b$ Plane Separating the Allowed from the Forbidden REGIONS

\begin{tabular}{lclll}
\hline \multicolumn{2}{c}{ Self-focusing } & & \multicolumn{2}{c}{ Self-defocusing } \\
\cline { 1 - 1 } Marginal Loci & Guided Power & & Marginal Loci & Guided Power \\
\hline$b \rightarrow b_{c+}$ & $\rightarrow 0$ & & $b \rightarrow b_{c+}$ & $\rightarrow 0$ \\
$b \rightarrow b_{c-}$ & finite & & $b \rightarrow 0$ & finite \\
$b \rightarrow b_{c l}, b_{c f}$ & $\rightarrow \infty$ & & $b \rightarrow b_{c d}$ & $\rightarrow \infty$ \\
\hline
\end{tabular}

solution is written as

$$
P=\frac{1}{2} N c \epsilon_{0} D E_{0}^{2} \int_{-\infty}^{+\infty} \Psi^{2}(\xi) d \xi
$$

where $c$ and $\epsilon_{0}$ are the speed of light in vacuo and the characteristic permitivity of free space, respectively. Using Langbein et al. formalism [8], this expression can be evaluated without knowledge of the explicit form of $\Psi(\xi)$. One gets,

$$
P=\frac{1}{2} N c \epsilon_{0} D E_{0}^{2}\left[I_{c}+I_{f}+I_{s}\right]
$$

with

$$
\begin{aligned}
I_{c}= & \frac{\Psi_{c}^{2}(\xi=0)}{2 V \sqrt{a+b}} \\
I_{f}= & \Psi_{c}^{2}(\xi=0)\left[\frac{1+A^{2}}{2}+\frac{1-A^{2}}{2} \frac{\sin (2 V \sqrt{1-b})}{2 V \sqrt{1-b}}\right. \\
& \left.+A \frac{\sin ^{2}(V \sqrt{1-b})}{V \sqrt{1-b}}\right]
\end{aligned}
$$

and

$I_{s}=\left[\int_{0}^{\Psi_{s, \max }}+(-1)^{M} \int_{\Psi_{s, \max }}^{\Psi_{s}(\xi=1)}\right] \frac{\Psi_{s}^{2}}{V \sqrt{\left[b-f\left(\Psi_{s}\right)\right] \Psi_{s}^{2}}} d \Psi_{s}$

where $A=\sqrt{(a+b) /(1-b)}$, and $\Psi_{s, \max }$ stands for the maximum value of $\Psi_{s}(\xi)$. According to the phase paths in Fig. 2, one has $\Psi_{s, \max }=\Psi_{s}(\xi=1)$ for pure guided waves, and $f\left(\Psi_{s, \max }\right)=b$ otherwise.

Let us start with a self-focusing medium. First, at the marginal loci $b \rightarrow b_{c+}, b_{c^{-}}$, one has $d=0$. Therefore, the field at the film-substrate interface, $\Psi_{s}(\xi=1)$, vanishes, and so does $\Psi_{c}(\xi=0)$. This yields $I_{c}=0$ and $I_{f}$ $=0$ for both loci. At $b_{c+}, I_{s}$ also vanishes, since so do the limits of the integral, whereas the function into the integral remains finite. Thus, $P=0$ at $b_{c_{+}}$, as it was expected. On the other hand, at $b_{c-}$ field solutions correspond to bulged guided waves, thus (21) becomes

$$
\left.I_{s}\right|_{b_{c-}}=2 \int_{0}^{\Psi_{s, \text { max }}} \frac{\Psi_{s}^{2}}{V \sqrt{\left[b_{c-}-f\left(\Psi_{s}\right)\right] \Psi_{s}^{2}}} d \Psi_{s} .
$$

At $\Psi_{s}=\Psi_{s, \text { max }}$ the function into the integral diverges, but since $f(\Psi)$ has been assumed to be a monotonic increasing function, the whole integral converges. So, we conclude that at the marginal loci $b \rightarrow b_{c}$ - the guided power amounts to a finite value, which will be different for any particular nonlinear permitivity $\epsilon^{\mathrm{NL}}$.

Concerning $b_{c l}$, which only occurs when strongly saturable media are considered $\left(f_{\max }<1\right)$, at this loci one has $d=f_{\max }$. This condition yields $\Psi_{s}(\xi=1) \rightarrow \infty$, and thus $\Psi_{c}(\xi=0) \rightarrow \infty$, also. Therefore, (19) and (20) diverges and we conclude that this loci cannot be reached by a finite value of the guided power. This conclusion holds also for $b_{c f}$. In this case, since $d<f_{\max }$, both $\Psi_{s}$ $(\xi=1)$ and $\Psi_{c}(\xi=0)$ amount to finite values, but $\Psi_{s, \max } \rightarrow \infty$. This yields the integral (21) to diverge and so does $P$.

When the nonlinear material forming the substrate exhibits a self-defocusing permitivity, only PGW are allowed, so (21) writes

$$
\left.I_{s}\right|_{\text {self-defocusing }}=\int_{0}^{\Psi_{s}(\xi=1)} \frac{\Psi_{s}^{2}}{\sqrt{\left[b+\left|f\left(\Psi_{s}\right)\right|\right] \Psi_{s}^{2}}} d \Psi_{s} .
$$

As above, in this case the curves $b_{c+}$ correspond to the low-power limit. On the other hand, at $b=0$ the electric field at both film-substrate and film-cover interfaces remain finite and so does (23). In conclusion, at $b \rightarrow 0$ the guided power amounts to a non-zero finite value. Finally, the loci $b=b_{c d}$ yield an analogous case as $b_{c l}$ did, in such a way that one gets $P \rightarrow \infty$ here again. These results drive us to the conclusion that the critical normalized thickness $V_{c d}^{(m)}$ separates the region in the parameter space where there is a maximum of the allowed guided power from the region where there is not.

In the Kerr-like limit, the locus $b_{c l}$ disappears and $b_{c f}$ goes to infinity, so for a self-focusing medium all allowed regions in the $V-b$ plane can be reached with a finite value of the guided power. Instead of this, in the case of a self-defocusing medium, $b_{c d}$ remains finite, thus above $V_{c d}^{(m)}$ the minimum value of $b$ is bounded by $b_{c d}$, although the guided power is not. The analytical expressions of the critical guided power at $b_{c-}$ (self-focusing medium) and $b=0$ (self-defocusing medium) for this limiting case can be found in [19].

\section{The Locus $d P / d \beta=0$}

The results obtained in previous sections enable us to find out the different kind of stationary nonlinear TE waves guided by a particular waveguide, as well as the range of possible values of the guided wave power, starting from $f_{\max }$ (or $f_{\min }$ ) and the values of the asymmetry measure and the normalized thickness of the waveguide. This information comes directly from the $V-b$ diagram and, keeping in mind the restrictions mentioned above, it does not depend on the particular expression of $\epsilon^{\mathrm{NL}}$. The other crucial information required, in order to characterize the stationary guiding properties of a given structure, is the relation between the guided power and the effective index of guided solutions. This relation does depend on the actual expression of $\epsilon^{\mathrm{NL}}$. Our aim in this section is to 
show that a great deal of this information can also be included in the corresponding $V-b$ diagram. We will restrict our attention to the $\mathrm{TE}_{0}$ guided wave and, for illustrative purposes, we will consider a self-focusing medium with a nonlinear dielectric permitivity given by [18]

$$
\epsilon^{\mathrm{NL}}=\epsilon_{\mathrm{sat}} \frac{u^{2}+2 u}{(u+1)^{2}}
$$

where $\epsilon_{\text {sat }}$ stands for the saturation value of the optically induced relative permitivity, $u=\alpha_{s} E^{2} / 2 \epsilon_{\text {sat }}$, and $\alpha_{s}=$ $\epsilon_{0} c n_{s}^{2} n_{2 s}$ is the nonlinear coefficient of the medium. In this case

$$
f_{\max } \equiv \gamma=\frac{\epsilon_{\text {sat }}}{n_{f}^{2}-n_{s}^{2}} .
$$

Concerning the $\mathrm{TE}_{0}$ wave, the dispersion relation $\beta-P$ displays two different patterns, the actual existence of one of them depending on the values of the various waveguide parameters. In a first category, the guided power increases monotonically with $\beta$ (so, there is an unique value of $\beta$ for each value of $P$ ). Conversely, in a second one, there is a region of $\beta$ values where $P$ decreases as $\beta$ grows (thus, multiple solutions for $\beta$ exist for a given value of $P$ ). When considering a waveguide belonging to the first group, one has $d P(\beta) / d \beta>0$ for any value of $\beta$, whereas otherwise the sing of this derivative depends on the value of $\beta$. Therefore, we conclude that the key point is to plot the locus where $d P(\beta) / d \beta=0$, in the $V$ $-b$ diagram.

For the following, it is worth to introduce a full set of normalized parameters describing the stationary guiding properties of the system we are dealing with. For convenience, according to [19], in addition to $b, V$, and $a$, let us use the normalized power measure $e_{s}=2 n_{s} n_{2 s} D k_{0}^{2} P$, the generalized aspect ratio between film and substrate refractive indexes $\delta_{n}=n_{f} / n_{s}$ and the saturation parameter $\gamma$ previously defined. In terms of these normalized variables, the condition $d P(\beta) / d \beta=0$ becomes

$$
\left.\frac{d e_{s}(b)}{d b}\right|_{b=b_{c c}}=0
$$

where $b_{c c}$ stands for the critical value of $b$ at which (26) holds. This equation defines an implicit function $b_{c c}=$ $\beta_{c c}\left(V, a, \delta_{n}, \gamma\right)$, which yields the locus to be plotted in the $V-b$ diagram corresponding to the asymmetry measure $a$, for given values of $\gamma$ and $\delta_{n}$. In Figs. 7 and 8 we have plotted the curve $b_{c c}(V)$ for $a=0$ and $a=10$, respectively. Shady regions indicate $\{V, b\}$ pairs for which the slope of the normalized dispersion curve $e_{s}(b)$ is negative. At the other pairs of allowed points in the $V-b$ plane, the slope of this curve is positive. Thus, it should be realized that for each given value of the normalized thickness of the waveguide, the shape of the dispersion relation $e_{s}(b)$ (and consequently $P(\beta)$ ) may be read-off immediately from $V-b$ diagram. In most practical applications the parameter $\delta_{n}$ makes only a slight influence on the curve $b_{c c}(V)$, particularly when considering asym-

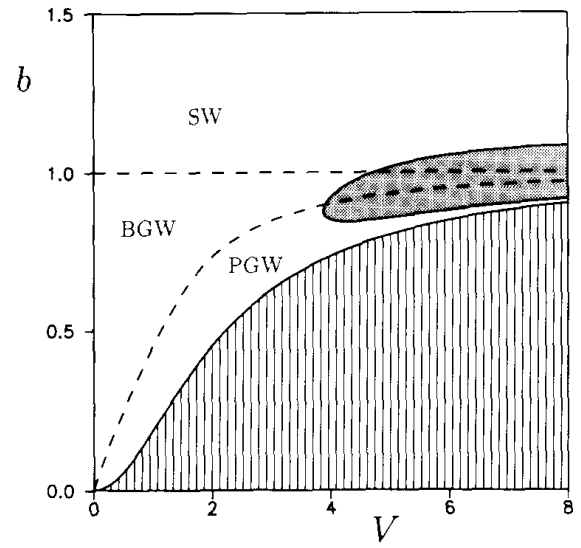

Fig. 7. Marginal locus $b_{c c}$ for the $\mathrm{TE}_{0}$ guided wave in a symmetric waveguide. Nonlinear permitivity given by (24), with $\gamma=2$. Shady region indicates $V-b$ pairs at which the guided power decreases with $\beta$. Here $\delta_{n}$ $=1.013$, so $b_{c r} \approx b_{c r}$

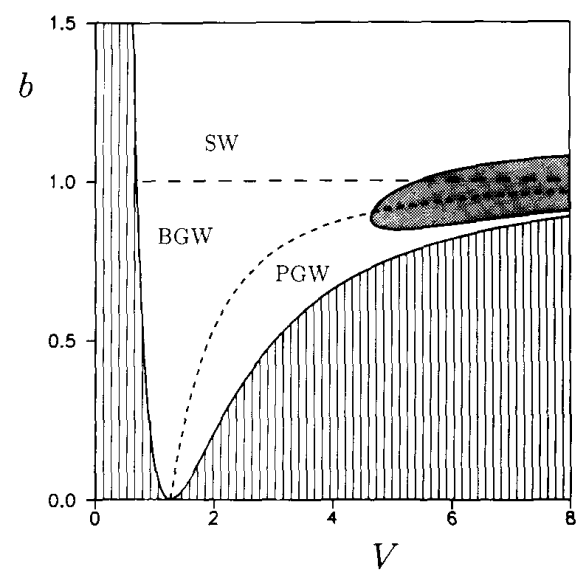

Fig. 8. Same as in Fig. 7 but for an asymmetric waveguide $(a=10)$.

metric structures. This statement comes from the fact that, out of the marginal loci $b_{c-}, b_{c f}$, and $b_{c l}$, this parameter only plays a relevant role when the factor $\left(\delta_{n}^{2}-1\right) b_{c c}$ amounts to values comparable or greater than the unity [19]. Concerning $\gamma$, it is obvious that $b_{c c}$ depends strongly on this parameter when $\gamma \sim 1$, whereas $b_{c c}$ is nearly insensitive to the actual value of $\gamma$ when $\gamma \gg 1$.

The loci $b_{c c}$ in Figs. 7 and 8 display an important feature: in both cases, there is a critical minimum value of the normalized thickness below which $b_{c c}$ does not exist, and so $e_{s}(b)$ is always an increasing function of $b$. This threshold value of $V$, which will be referred to as $V_{c c}$, is obtained from the formal expression

$$
\left.\frac{d b_{c c}(V)}{d V}\right|_{V=V_{c i}} \rightarrow \infty
$$

Since $b_{c c}=\Theta_{c c}\left(V, a, \delta_{n}, \gamma\right)$, we conclude that $V_{c c}=$ $\nabla_{c c}\left(a, \delta_{n}, \gamma\right)$. Note that when considering asymmetric waveguides with $\gamma>1$, one has $V_{c c} \approx V_{c c}(\infty) \equiv \nabla_{c c}(a$ 
$\left.\rightarrow \infty, \delta_{n} \rightarrow 1, \gamma \rightarrow \infty\right)$. The outstanding point to be emphasized is that this is a universal quantity. Numerical calculations yield $V_{c c}(\infty) \simeq 3.65$.

Finally, the locus $b_{c c}$ seems to be of great significance concerning another crucial point: the stability of the $\mathrm{TE}_{0}$ nonlinear guided wave supported by the waveguide we are analyzing. Up to now, to the best of our knowledge, a stability criterion for nonlinear waves propagating in an optical waveguide with a saturable substrate or cladding is still lacking. However, after several investigations [24][26] there is an important numerical evidence supporting the hypothesis that the $\mathrm{TE}_{0}$ guided wave is stable in the regions of the dispersion curves where

$$
\frac{d}{d N} \int_{-\infty}^{\infty} \Psi^{2}(\xi) d \xi \equiv \frac{d I(N)}{d N}>0
$$

and unstable on negatively sloped branches. This is the conclusion which has been also established in the Kerrlike limit [27]-[30]. Thus neutral stable waves would correspond to $d I(N) / d N=0$. In terms of the normalized effective index this equation becomes

$$
\left.\frac{d I(b)}{d b}\right|_{b=b_{c r}}=0
$$

$b_{c r}$ being just defined by this expression. This equation involves only the parameters $b, V, a$, and $\gamma$, therefore it defines an implicit function $b_{c r}=\wp_{c r}(V, a, \gamma)$, which would yield the locus of marginal linear stability in the $V$ $-b$ diagram. In fact, in the formal limiting $\delta_{n} \rightarrow 1,(34)$ coincides with (30), therefore $b_{c r}=b_{c c}\left(\delta_{n} \rightarrow 1\right)$. In Figs. 7 and $8, \delta_{n} \approx 1$, so $b_{c r}$ and $b_{c c}$ cannot be distinguished from each other. Thus, according to the above mentioned conjecture we shall conclude that: 1) shady regions in Figs. 7 and 8 would indicate unstable waves; 2) the other allowed regions in the $V-b$ plane would correspond to stable $\mathrm{TE}_{0}$ waves; 3$) b_{c r}=b_{c c}\left(\delta_{n} \rightarrow 1\right)$ would be the locus of marginal or neutral linear stability; 4) below $V_{c r}=$ $V_{c c}\left(\delta_{n} \rightarrow 1\right)$ all $\mathrm{TE}_{0}$ guided waves would be stable to propagation along the nonlinear waveguide.

\section{Concluding Remarks}

The main conclusion of this paper is that universal $V$ $-b$ diagrams provide a very powerful tool when describing the stationary waveguiding properties of the TE waves guided by a thin film bounded by a saturable nonlinear substrate or cladding. We have shown that, for a wide class of nonlinearities, the allowed and forbidden regions of the $V-b$ plane for stationary guided propagation to occur display a universal pattern, the marginal loci separating different allowed regions (for $\mathrm{PGW}, \mathrm{BGW}$, and SW) from the forbidden ones being a function of only the waveguide asymmetry measure and the saturation value of the nonlinear permitivity.

Starting from the explicit expressions of these marginal curves, we have been able to obtain power-independent critical threshold values of the waveguide normalized thickness for each particular kind of stationary nonlinear guided wave to be allowed. Marginal loci yield also the asymptotical values of the normalized effective index for large values of the guided power flow, which are independent on the particular form of $\epsilon^{\mathrm{NL}}$ as well. Qualitative information concerning the dispersion relation $\beta-P$ can be also included in the diagrams, plotting on them the critical locus where $d P(\beta) / d \beta=0$. In the limiting $\delta_{n} \rightarrow$ 1 , this locus coincides with $d I(\beta) / d \beta=0$, which seems to be the locus of marginal linear stability for the $\mathrm{TE}_{0}$ guided waves, so then stability of such guided solutions supported by a particular waveguide can be also read off from its $V-b$ diagram. Other nonlinearities than the ones treated here, as well as more complicated structures, can be also considered.

Finally, the value of $f_{\max }$ (or $f_{\min }$ ) has been shown to give the figure of merit of a particular waveguide, concerning saturation effects: $\left|f_{\max , \min } \ll 1\right|$ corresponds to the strong saturation regime, whereas $\left|f_{\max , \min }\right| \gg>1$ yields the nearly Kerr-like behavior. Let us focus on the particular permitivity (24), thus $f_{\max }=\gamma$, which is illustrative of a typical two-level response. According to Figs. 3 and 4 , for highly nonlinear guided waves to exist, large values of $\gamma$ are required. Liquid crystals are known to exhibit such large nonlinearities, allowing observation of intensity-dependent guided waves [21] and switching [31], although response time limitates their usefulness for device applications. Semiconductor-doped glasses, GaAsAlGaAs MQW's and organic materials avoid this difficulty, but with smaller $\epsilon_{\text {sat }}$. In usual waveguiding structures this yields very small values of $\gamma$ : tipically $\gamma \sim 10^{-2}$ in a GaAs-AlGaAs MQW near half gap. This has been shown to be large enough for all-optical switching, but is by far too small for observation of nonlinear guided waves in conventional waveguides made on these materials. Larger values of $\epsilon_{\mathrm{sat}}$ will increase $\gamma$, but the point is that this goal can be also achieved using waveguides with smaller $\left(n_{f}-n_{s}\right)$. In fact, $\gamma$ states the measure of this (otherwise) intuitive rule. In this line, we quote recent work by Lederer and co-workers [32], which indicates that strongly nonlinear waves could also be realized by using properly designed waveguiding structures, yielding effective waveguides with $\left(n_{f}-n_{s}\right) \ll 1$, made on weakly nonlinear materials.

\section{REFERENCES}

[1] N. N. Akhmediev, "Novel class of nonlinear surface waves: asymmetric modes in a symmetric layered structure," Sov. Phys. JETP, vol. 56, pp. 299-303, 1982 .

[2] F. Lederer, U. Langbein, and H.-E. Ponath, "Nonlinear waves guided by a dielectric slab," Appl. Phys. B, vol. 31, pp. 69-73, 1983.

[3] U. Langbein, F. Lederer, H.-E. Ponath, and U. Trutschsel, "Dispersion relations for nonlinear guided waves," J. Molec. Struc, , vol. 115, pp. 493-496, 1984.

[4] A. Boardman and P. Egan, "Theory of optical hysteresis for TE guided modes,"' Phil. Trans. R. Soc. London A, vol. 313, pp. 363369,1984 .

[5] D. Mihalache, D. Mazilu, and H. Totia, "Bistable states of s-polarized nonlinear waves guided by an asymmetric three-layer dielectric structure," Phys. Scr., vol. 30, pp. 335-340, 1984. 
[6] A. D. Boardman and P. Egan, " $S$-polarized waves in a thin dielectric film asymmetrically bounded by optically nonlinear media," IEEE $J$. Quantum Electron., vol. QE-21, pp. 1701-1713, 1985.

[7] G. I. Stegeman and C. T. Seaton, "Nonlinear integrated optics," $J$. Appl. Phys., vol. 58, pp. R57-78, 1985.

[8] U. Langbein, F. Lederer, and H.-E. Ponath, "Generalized dispersion relations for nonlinear slab-guided waves," Opt. Commun., vol. 53 , pp. 417-420, 1985.

[9] G. I. Stegeman and E. M. Wright, "All-optical waveguide switch ing," Opt. Quantum Electron., vol. 22, pp. 95-122, 1990

[10] S. M. Jensen, "The nonlinear coherent coupler," IEEE J. Quantum Electron., vol. QE-18, pp. 1580-1583, 1982

[11] X. J. Meng and N. Okamoto, "Improved coupled-mode theory for nonlinear directional couplers," IEEE J. Quantum Electron., vol. 27, pp. 1175-1181, 1991 .

[12] D. R. Heatley, E. M. Wright and G. I. Stegeman, "Soliton coupler," Appl. Phys. Lett., vol. 53, pp. 172-174, 1988

[13] C. T. Seaton, Xu Mai, G. I. Stegeman, and H. G. Winful, "Nonlinear guided wave applications," Opt. Eng., vol. 24, pp. 593-599, 1985

[14] G. I. Stegeman, E. M. Wright, C. T. Seaton, J. V. Moloney, T.-P. Shen, A. A. Maradudin, and R. F. Wallis, "Nonlinear slab-guided waves in non-Kerr-like media," IEEE J. Quantum Electron,, vol. QE-22, pp. 977-983, 1986.

[15] G. I. Stegeman, J. Ariyasu, C. T. Seaton, T. P. Shen and J. V. Moloney, "Nonlinear thin-film guided waves in non-Kerr media," Appl. Phys. Lett., vol. 47, pp. 1254-1256, 1985.

[16] D. Mihalache, D. Mazilu, M. Bertolotti, and C. Sibilia, "Exact solution for nonlinear thin-film guided waves in higher-order nonlinear media," J. Opt. Soc. Amer. B, vol. 5, pp. 565-570, 1988.

[17] U. Langbein, F. Lederer, T. Peschel, and H.-E. Ponath, "Nonlinear guided waves in saturable nonlinear media," Opt. Lett., vol. 10, pp. 571-573, 1985.

[18] V. E. Wood, E. D. Evans, and R. P. Kenan, "Soluble saturable refractive-index nonlinearity model," Opt. Commun., vol. 69, pp. 156$160,1988$.

[19] L. Torner and J. P. Torres, "Similarity rules for nonlinear Kerr-like slab optical waveguides," IEEE J. Quantum Electron., vol. 28, pp. $1571-1581,1992$

[20] H. Kogelnik and V. Ramaswamy, "Scaling rules for thin-film optical waveguides," Appl. Opt., vol. 13, pp. 1857-1862, 1974

[21] H. Vach, C. T. Seaton, G. I. Stegeman, and I. C. Khoo, "Obser vation of intensity-dependent guided waves," Opt. Lett., vol. 9, pp. $238-240,1984$.

[22] G. I. Stegeman and R. H. Stolen, "Waveguides and fibers for nonlinear optics," J. Opt. Soc. Amer. B, vol. 6, pp. 652-662, 1989

[23] G. I. Stegeman, A. Villeneuve, P. Wigley, C. C. Yang, C. Ironside, S. Aitchison, and A. H. Kean, "Nonlinear optical interactions in AlGaAs near half the band gap," in OSA Tech. Dig., vol. 13, pp. 106-108, 1992, QELS'92 paper QTuN3.

[24] D. Mihalache and D. Mazilu, "Stability of nonlinear stationary slab guided waves in saturable media: a numerical analysis," Phys. Lett. $A$, vol. 122 , pp. $381-384,1987$.

[25] _ " "Stability and instability of nonlinear guided waves in saturable media," Solid State Commun., vol. 63, pp. 215-217, 1987.
[26] M. Gubbels, E. M. Wright, G. I. Stegeman, C. T. Seaton, and J. V Moloney, "Effects of absorption on the $\mathrm{TE}_{0}$ nonlinear guided waves," Opt. Commun., vol. 61, pp. 357-362, 1987

[27] N. N. Akhmediev, V. I. Korneev, and Yu. V. Kuz'menko, 'Excitation of nonlinear surface waves by Gaussian light beams," Sov. Phys. JETP, vol. 61, pp. 62-67, 1985.

[28] C. K. R. T. Jones and J. V. Moloney, "Instability of standing waves in nonlinear optical waveguides," Phys. Lett. A, vol. 117, pp. 175$180,1986$.

[29] L. Leine, C. Wächter, U. Langbein, and F. Lederer, "Evolution of nonlinear guided optical fields down a dielectric film with a nonlinear cladding, "J. Opt. Soc. Amer. B, vol, 5, pp. 547-558, 1988.

[30] D. Mihalache, M. Bertolotti, and C. Sibilia, "Nonlinear wave prop agation in planar structures," in Progress in Optics, vol. 27, E. Wolf, Ed. Amsterdam: North-Holland, 1989, pp. 227-313.

[31] I. C. Khoo, P. Zhou, R. R. Michael, R. G. Lindquist, and R. Mansfield, "Optical switching by a dielectric-cladded nematic film," IEEE J. Ouantum Electron., vol. 25, pp. 1755-1759, 1989.

[32] F. Lederer, U. Trutschel, T. Peschel, Ch. Wächter, and A. D. Boardman, "Large nonlinear effects with weak nonlinearities in properly tailored waveguides," in OSA Tech. Dig., vol. 9, pp. 430-432, 1992, IQEC'92 paper PTh026.

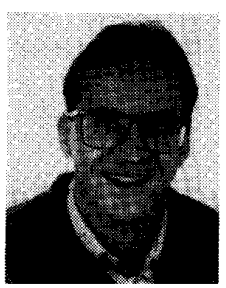

Juan P. Torres was born in Barcelona, Spain in 1964. He received the B.S. degree in physics from the University of Barcelona in 1987

In 1991 joined the School of Telecommunication Engineering of the Polytechnic University of Catalonia where he is currently pursuing the Ph.D. degree in science. His research interest is on nonlinear guided waves optics and its applications.

Mr. Torres is a student member of the IEEE Laser and Electro-Optics Society and of the Optical Society of America.

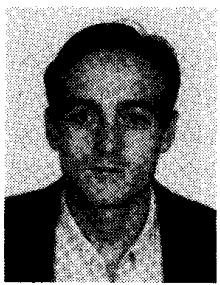

Lluís Torner (M'92) was born in Barcelona, Spain on September 20,1961. He received the B.Sc. and M.Sc. degrees in physics from the $\mathrm{Au}$ tonomous University of Barcelona in 1985 and 1986, respectively, and the $\mathrm{Ph} . \mathrm{D}$. degree in engineering science in 1989, from the Polytechnic University of Catalonia, Barcelona.

He is currently an Associate Professor with the Department of Signal Theory and Communications, Polytechnic University of Catalonia, Barcelona. He started his research activities in the field of Rayleigh and Brillouin scattering. His current major interests include integrated optics, nonlinear optical waveguide phenomena, all-optical switching, and integrated optical sensors.

Dr. Torner is a member of the IEEE Lasers and Electro-Optics Society, the Optical Society of America, and the European Optical Society. 